\title{
TRAZANDO LA FRONTERA ARAUCANA, CHILE. De las leyes y las divisiones del territorio. 1866-1916
}

\author{
Barria Catalán, Tirza Aseneth \\ Universidad Austral de Chile \\ tirzabarria@uach.cl
}

\section{RESUMEN}

Con la "Pacificación de la Araucanía", se inició en la segunda mitad del s.XIX, un proceso que cambió la fisonomía del territorio llamado "frontera araucana". La propiedad privada y pública se mensuró, delimitó y fijó en planos, determinándose la radicación mapuche, y el remate y entrega de tierras a colonos nacionales y extranjeros. Del análisis de planos y en especifico de la "Carta Jeneral de Colonización de la provincia de Cautín", elaborada por Nicanor Boloña e impresa en 1916, y su relación con la Ley de 1866, este articulo tiene como objetivo visibilizar el proceso de territorialización de la frontera. Por medio del redibujo de la "carta", se elaboran una serie de mapas temáticos entre ellos los de hidrografía, infraestructura, urbanos, administrativos y de parcelación. Se observa en la macro escala una continuidad del espacio territorial dado por el trazado de hijuelación, formas determinadas por la ley de 1866 y sus sucesivas leyes complementarias.

Palabras clave: colonización, frontera, hijuelación, cartografía Bloque temático: Análisis y proyecto territorial

\begin{abstract}
With the "Pacification of Araucanía", a process that changed the physiognomy of the territory called "Araucana border" began in the second half of the 19th century. Private and public property was measured, delimited and fixed in plans, determining the Mapuche settlement, and the auction and delivery of land to national and foreign settlers. From the analysis of plans and specifically the "Jeneral Letter of Colonization of the province of Cautín", prepared by Nicanor Bologna and printed in 1916, and its relationship with the Law of 1866, this article aims to make visible the territorialization process of border. Through the redrawing of the "letter", a series of thematic maps are prepared, including those of hydrography, infrastructure, urban, administrative and partitioning. The continuity of the territorial space given by the plotting of the land, the forms determined by the law of 1866 and its successive complementary laws is observed in the macro scale.
\end{abstract}

Keywords: colonization, border, hybrids, cartography

Topic: Analysis and territorial project 


\section{1.- Introducción}

Cada territorio es una mezcla específica de diversas componentes, sean estas morfológicas o tejidas por acción humana. De la disposición de caminos, de los lugares de cruce y de intercambio, de la construcción de infraestructura, de las formas de la propiedad, y el emplazamiento de las industrias y las ciudades, se identifica una historia de ocupación, cuya dinámica está sostenida por intereses políticos, capitalistas o de otra índole (Parcerisa y Busquets, 1983).

En este contexto los mapas son producidos como un dispositivo ideológico que fijó limites y dominio sobre los "espacios vacíos" del territorio, donde la racionalización, delimitación y ocupación de este, fueron prácticas llevadas a cabo en las tierras de frontera. Estos procesos de colonización impulsados por diversos países, se observan en Estados Unidos, Nueva Zelanda, Brasil y Argentina (Jackson Turner, 1986; Favelukes, 2013) que, al amparo de diversos objetivos, buscaron medir, integrar y catastrar el territorio ocupado, y ejercer soberanía y control sobre las futuras tierras productivas. Tales transformaciones implicaron no solo el desplazamiento gradual de la línea de frontera hasta su eliminación, sino también cambios radicales en la fisonomía del paisaje y en la relación de sus habitantes con su territorio.

En el sur de Chile, se eliminaron las antiguas fronteras de la zona mapuche, territorio comprendido entre los ríos Biobío por el norte y, Toltén por el sur (Méndez, 1982; Villalobos, 1982) como resultado de la puesta en marcha de la "Pacificación de la Araucanía", estrategia de ocupación llevada a cabo por el Ejército de Operaciones de Frontera entre 1866 y 1883, desencadenándose un proceso de ocupación y explotación agrícola de las "nuevas tierras" disponibles del Estado (Saavedra, 1870; Guevara, 1902; Navarro, 1909).

La colonización de esta zona, implicó la puesta en práctica de distintas modalidades de ocupación, todas amparadas en un cuerpo legal de reglamentos y decretos elaborados desde 1866 (Aylwin, 1995). Junto a ello, se crearon la Oficina General de Inmigración con el propósito de formentar la colonización de la zona y, la Sociedad Nacional de Agricultura, cuya función fue la de preparar los lotes destinados a los colonos, y entregar los materiales y objetos y necesarios para su asentamiento. Por su parte, la Sección Topográfica, de la Inspección de Tierras y Colonización, del Ministerio de Relaciones Exteriores, Culto y Colonización (18881924), elaboró una serie de planos de loteo necesarios para el remate de tierras en subasta pública y otros, que delimitaron la propiedad mapuche para la radicación indígena (Álvarez, 2014).

Si bien las instituciones del Estado propiciaron la regulación legal en la entrega de lotes, la situación no siempre resultó como dictaba la ley. Este problema quedó en evidencia en la investigación que llevó a cabo la Comisión Parlamentaria de Colonización, quienes en 1912 informaron de las irregularidades de los procedimientos y la falta de claridad y aplicación de estos como así de los actos indebidos de los ocupantes y colonos, de los vacíos de las leyes vigentes y de las deficiencias en su aplicación. En consecuencia, la Inspección General de Colonización y a la Oficina de Mensura de Tierras procedieron a determinar los terrenos que disponía el Estado, especificando su extensión y deslindes, las ocupaciones ya autorizadas y las que tuvieran por base un título regular. Ello implicó, disponer de todos los registros de planos de mensura y de las mediciones de las tierras fiscales, de manera de contar con un panorama general del proceso de colonización de la Araucanía. 


\section{2.- Del registro cartográfico. El "Plano de 1908" y la "Carta de 1916"}

Bajo el mandato presidencial de Pedro Montt (1906-1910) se creó la Oficina de Mensura de Tierras (1907), la cual se formó de la unión de la Oficina de Límites, la Sección Topográfica dependiente de la Inspección de Tierras y Colonización y, la Comisión Radicadora de Indígenas. El objetivo de esta oficina fue la de desempeñar las labores de cartografía y demarcación de la propiedad fiscal de los territorios destinados a la colonización, actividad denominada hijuelación, la cual comprendía la ejecución del plano y la entrega a través de arriendo, venta o concesión a los interesados. Además, tenía a su cargo la tarea de resguardar las tierras fiscales sin ocupación, lo que representaba tener a su custodia la propiedad estatal, con la finalidad de protegerla de ocupaciones ilegales. Se suma a lo anterior, el resguardo de documentos como planos de las tierras del sur, las actas de entrega de estas y, en resumen, todos los títulos y documentos que se refieren a la constitución de la propiedad fiscal en las provincias australes (Álvarez, 2014).

De esta época data, el primer Plano levantado para la provincia de Cautín en 1908. El mapa identificado como "Plano Jeneral de la rejión comprendida entre los ríos Cautín, Imperial i Toltén, entre línea férrea y Océano Pacifico, formado con los planos particulares levantados en esta zona", a escala 1:50.000, comprende la mitad del territorio de la provincia de Cautín (fig.1). Se conforma de la unión de varios planos parciales y presenta una visión general en cuanto a las formas de división predial tanto de los mapuches, de colonos y de las concesiones otorgadas a las empresas de colonización. Este plano impreso en Temuco y donde lo único que se distingue de la firma es el apellido Navarrete, fue la base para la elaboración de un segundo gran mapa fechado en 1916.

Ocho años después, aparece la "Carta Jeneral de Colonización de la provincia de Cautín" (fig.2), confeccionada a escala 1:100.000, impresa en Santiago en los Talleres del Estado Mayor del Ejército, y firmada por Nicanor Boloña. En este mapa - en adelante "Carta de 1916" - se dibuja el total de territorio que abarca administrativamente para aquella época la provincia de Cautín delimitada en $1887^{1}$. Se dibujan los componentes esenciales de la geografía física como los cursos de aguas y el relieve de los cordones montañosos. Así también se identifican los trazados del ferrocarril y caminos, ejes de extensión que constituyen el primer signo de territorialización y, la división administrativa en departamentos, y subdelegaciones.

Otros antecedentes corresponden a las formas de división predial, que al igual que el plano de 1908, dan cuenta de modelos de trazados diferenciados, evidentes en la macro escala territorial y en su condición de conjunto. Se reitera el hecho de esta visión totalizadora de la provincia, es posible por la unión de varios planos parciales elaborados desde 1880, los cuales son la evidencia de un sistema de repartición de tierras ya sea de "iure" (por derecho) o de "facto" (por hechos). Esto quiere decir que, el trazado de mensura estuvo condicionado ya sea por el derecho de ocupación ancestral, situación que se dio en el caso de los títulos de merced o, por la "inexistencia" de habitantes, lo que determinó la imposición de un nuevo trazado divisible que rentabilizaba y facilitaba el remate público de tierras. Por consiguiente, la "Carta de 1916" es el resultado de la compilación de otros registros, y solo puede dar cuenta de una territorialización avanzada, cuyo proceso se había iniciado 50 años antes.

\footnotetext{
${ }^{1}$ La provincia de Malleco quedó enmarcada por el río Renaico por el norte y el río Cautín por el sur, siendo su capital Angol y la provincia de Cautín se emplazó entre el río Cautín por el norte y el río Toltén por el sur, cuya capital fue establecida en la ciudad de Temuco. Ver en González, J. \& Bermedo, P. Cartografía de la transformación de un territorio: La Araucanía 1852-1887. Santiago, Chile: Revista de Geografía Norte Grande, N $54,2013$.
} 


\section{SIIU}

barcelona|santiago dechile junio 2019

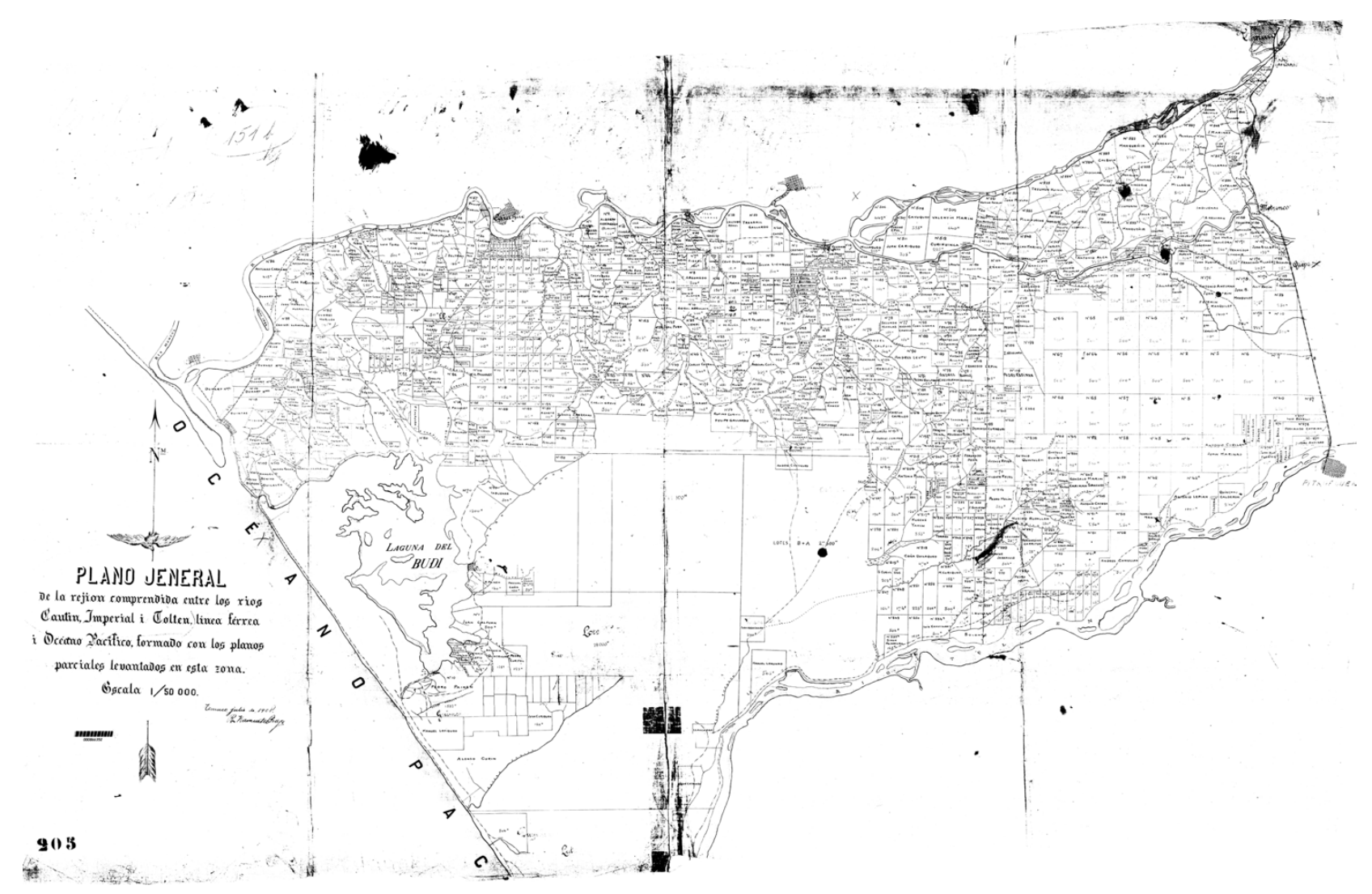

Fig 1"Plano jeneral de la región comprendida entre los ríos Cautín, Imperial i Toltén, línea férrea i océano Pacífico, formado con los planos parciales levantados en esta zona". Escala 1:50.000. Con fecha julio 1908, Temuco. Dibujo R, Navarrete. Fuente: http://www.catastro.cl/tmp/obj_94390/97989_000866352.pdf

La "Carta de 1916" de Cautín corresponde a un documento compuesto de 15 hojas de 49 por 32 centímetros. En su conjunto se identifican los cursos de aguas, el relieve de los cordones montañosos, la extensión ferroviaria y de caminos, la tenencia, numeración y dimensión de lotes y, la división administrativa de departamentos y subdelegaciones. También se reconoce el trazado urbano de 23 ciudades y villas las cuales, en su mayoría estaban conectadas a la red ferroviaria que se extendía desde Santiago y ubicadas a razón de 40 kilómetros entre ellas. Esta carta fue elaborada por Nicanor Boloña, cartógrafo de la Sección Topográfica de la Inspección General de Tierras y Colonización quien, al año siguiente publica su carta gemela para la provincia de Malleco, bajo los mismos parámetros de dibujo que la de Cautín de 1916 (fig. 2)

En esencia la "Carta de 1916" visibiliza un catastro del territorio colonizado, donde las formas de división del suelo apuntaban a la regularidad, articulación y continuidad del espacio territorial. Se identifican las condiciones topográficas que definieron los límites de la hijuelación, como también la forma regular del trazado ortogonal, el cual permitió por adición, la concentración de grandes extensiones en tierras productiva en pocos propietarios en su mayoría nacionales. Se reconoce también en el dibujo, el emplazamiento de colonos extranjeros preferentemente en los bordes de ríos bajo la lógica de lote alargado y, el trazado existente y futura de la infraestructura ferroviaria que condicionó el crecimiento de la ciudad. 


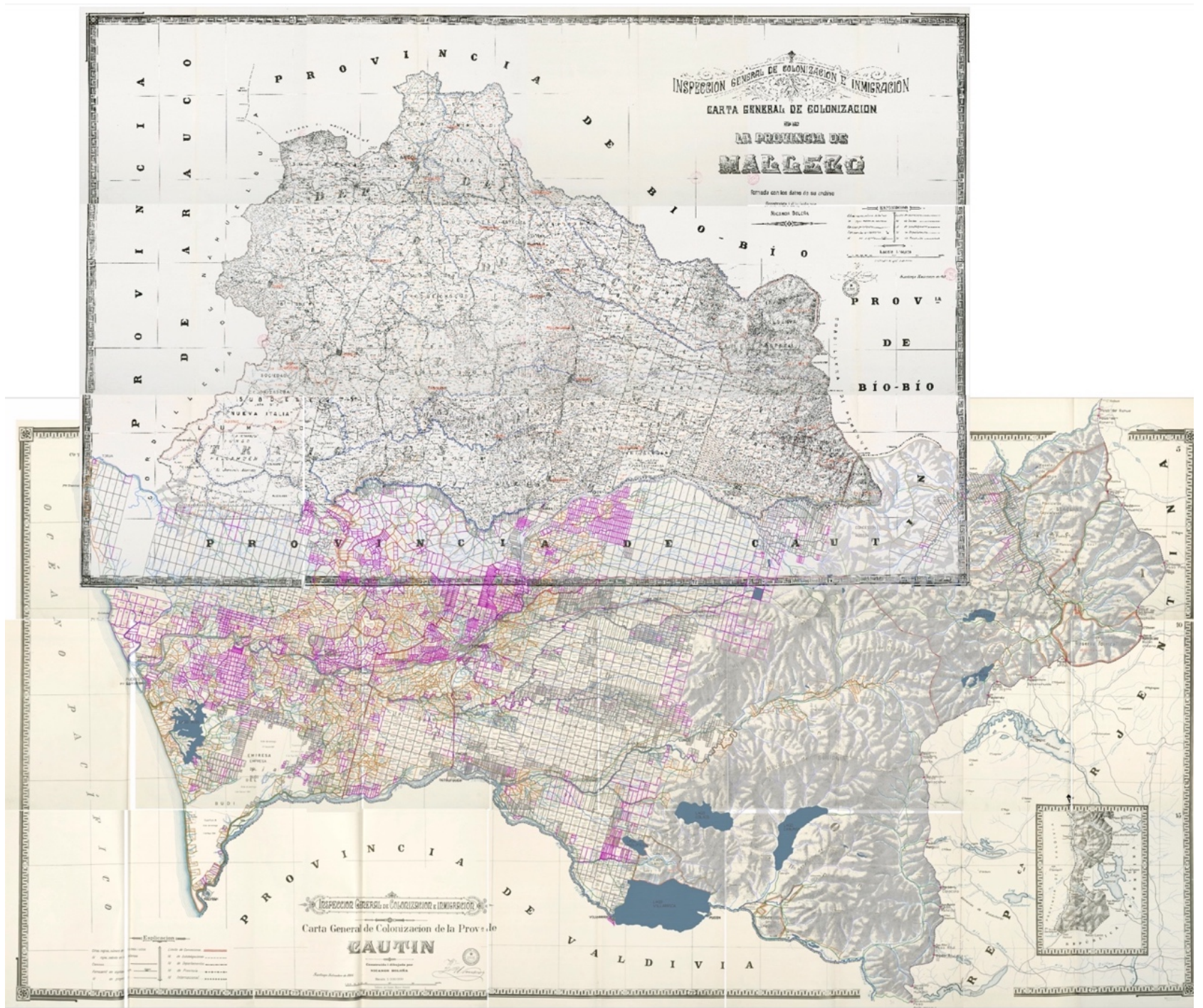

Fig. 2. Ensamble de las Carta de Cautín y Malleco. Fuente: Elaboración propia a partir de los Cartas disponible en el Archivo Histórico de la Araucanía.

Del análisis de ambas piezas cartográficas, la de 1908 y la de 1916, se observa un proceso de rectificación de lotes y actualización de la propiedad, sobre todo en la zona de la Concesión Budi. Otros aspectos a observar en la carta de 1916 y que no aparecen en la de 1908, es la información detallada de las propiedades dando cuenta del tamaño de hectáreas, numeración y nombre del propietario. 


\section{3.- Representación totalizadora. Continuidad espacial}

La "Carta de 1916" expone lógicas de división y modulación geométrica y orgánica donde las operatorias de trazado tenían como objetivo desagregar el territorio y escalar la extensión geográfica a partir de una visión totalizadora (fig. 3). Es así como se observan las distintas formas de división que tomaron los lotes los cuales conformaron un tejido diverso.

\section{Ciudades y poblados}

Las acciones para el poblamiento y ordenamiento del territorio en el siglo XIX, toma como protagonista a la ciudad, la cual fue el elemento articulador y configurador de las políticas de territorialización. En este sentido la ciudad, se constituyó como una estrategia de poder y dominio que colaboró en configurar un tipo de orden y sentido territorial.

Los trazados urbanos de las ciudades de la Araucanía recogen la lógica fundacional del damero que varía en orientación, esto quiere decir que algunos trazados de ciudades se rigen por el norte magnético. Por ejemplo, la ciudad de Temuco, fundada como fuerte en 1881 por Manuel Recabarren, ubicada en el centro de la provincia al margen del río Cautín presenta un trazado regular de damero, flanqueado al poniente por una diagonal, eje propuesto en el trazado del primer plano de 1892, elaborado por Teodoro Schmidt. Otros poblados como Galvarino, fundado como fuerte en 1882, y demarcado en 1886 por los ingenieros de colonización en base al norte geográfico, fue emplazado al margen izquierdo del río Quillén, al norte de la provincia.

A diferencia de los trazados anteriores de ciudades tipo damero, se elaboraron otros esquemas urbanos los cuales correspondieron a influencias derivadas de nuevas formas de urbanización. Ejemplo de ello es Villa Portales (actual Lonquimay) fundada en 1897 por Gregorio Urrutia en la zona cordillerana cuyo trazado corresponde a tres elipses concéntricas que forman sus calles de circunvalación principal o Pitrufquén (antigua Lisperguer) fundada en 1882, ubicada el curso medio del río Toltén en el límite sur de la provincia en cuyo trazado convergen cuatro calles diagonales hacia una plaza central.

\section{Conexiones viales y ferroviarias}

De la lectura total de la "Carta de 1916" se identificaron trazados de ferrocarriles y caminos, ejes de comunicación y conexión, con el propósito de colonizar un territorio ex novo. El sistema está compuesto por tres estructuras que aseguran y permiten el desplazamiento: una red de caminos, la ubicación de pasos fronterizos y una red ferroviaria con sus respectivas estaciones y bodegas. La red caminera se despliega desde la cordillera de Los Andes hasta el océano Pacifico, cruzando transversal y longitudinalmente el territorio. Se concentran particularmente en los valles que se forman entre los ríos Cholchol, Cautín y Quepe, y conecta todas ciudades fundadas y estaciones de trenes. 


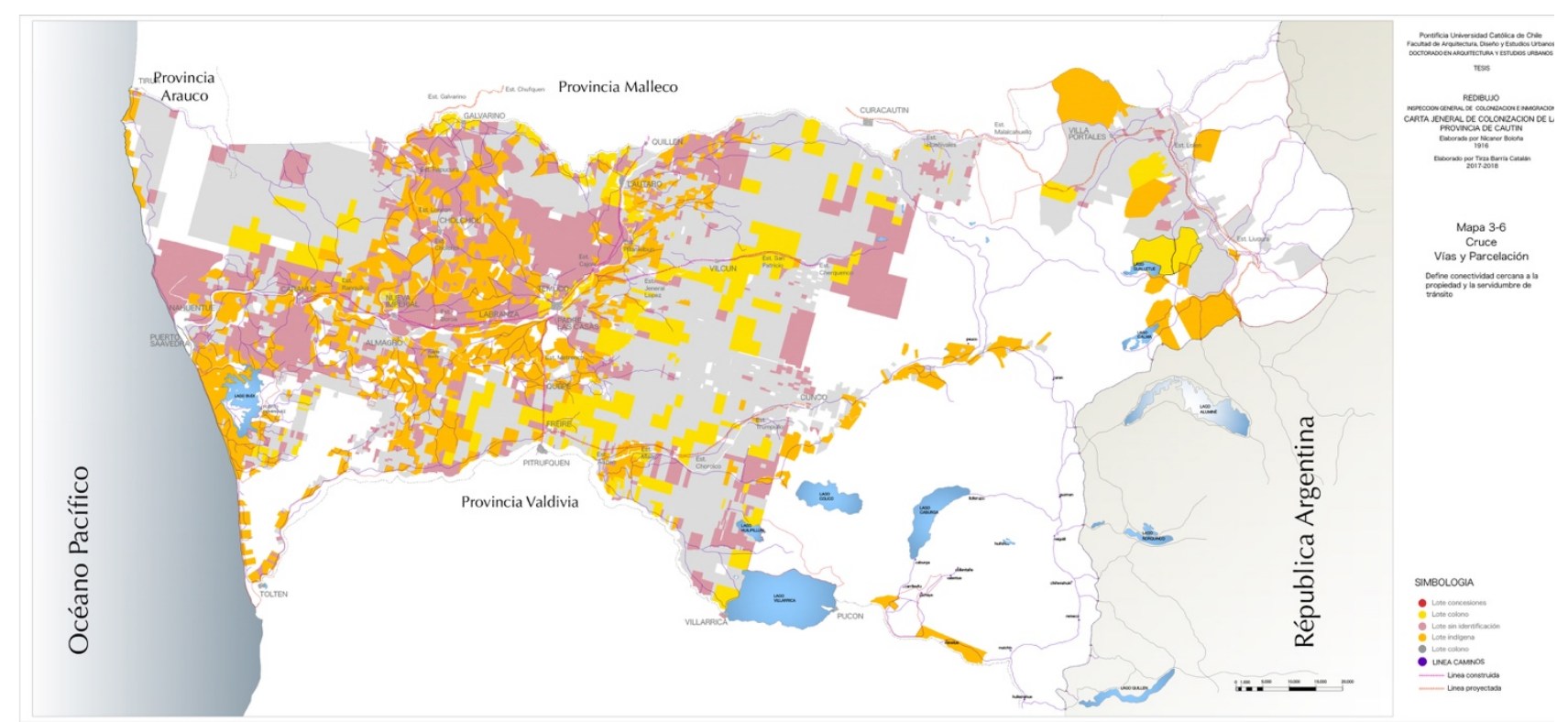

fig. 3.- Cruce de trazados de infraestructura, trazado urbano y lotes. Fuente: Elaboración propia a partir de los Cartas disponible en el Archivo Histórico de la Araucanía.

\section{Múltiples trazados. La hijuelación.}

El redibujo de la "Carta de 1916" (fig.4 y 5) permite identificar las diversas formas de los trazados de lotes (regulares y irregulares) como también la situación de la propiedad pública y privada coloncádola en relación con la infraestructura cial y ferroviara. Ejemplo de ello son el modelo de trazado que se sobrepone al territorio y sus componentes (hídrica y relieve). Estos trazados ortogonales, son lotes cuyo tamaño varía entre las 200 y las 500 hectáreas, y se orientan regularmente hacia el norte magnético. Se concentran preferentemente, de norte a sur, entre el curso del río Cautín y la pre cordillera de los Andes y, también al norte de la provincia entre el río Cholchol y la costa del Pacífico. si bien existió un procedimiento que regularizó el dimensionamiento y entrega de lotes para los colonos nacionales y extranjeros, queda en evidencia que las leyes no establecieron restricción alguna para que estos, adquirieran a través de remates de tierras fiscales, la cantidad de lotes que quisieran, generándose en estas zonas la concentración de tierras en pocas manos, fuese por razones de producción o especulación. Ello implicó que grandes propietarios de tierras registraran bajo su propiedad extensiones conformándose latifundios.

Otros trazados que responden a la ortogonalidad, son los inferiores a 200 hectáreas los cuales se concentran preferentemente en los cursos del río Huichahue y el río Quepe, y la extensión hacia el sur del río Cautín a la altura de la ciudad de Lautaro. Por el norte se emplazan al sur el río Cautín a altura de la ciudad de Curacautín y, también se concentran en los límites de la Concesión Empresa del Budi al sur-poniente de la provincia. Se suman a este grupo los trazados que colindan con curso de aguas. Estos corresponden a lotes asociados a radicaciones indígenas, de ahí su forma orgánica en el sentido que reconoce los accidentes geográficos que determinan su forma. Sin embargo, existen lotes de colonización extranjera distribuidas en torno al río Quillén por el norte y el borde del río Cautín entre las ciudades de Lautaro y Temuco. De estos asentamientos es característico la forma de lote alargada y perpendicular al curso del río, situación que permitió que usufructuaran de su caudal.

Otros tipos de mensura de tierra corresponden a los trazados irregulares donde sus límites están determinados, en la mayoría de los casos, por los cursos de agua, el relieve o incluso los caminos. Corresponden a lotes 
definidos como radicaciones indígenas y su tamaño varía de 30 hectáreas a 200 hectáreas. Estos se concentran principalmente entre los ríos Cholchol y Cautín, también hacia el norte de la provincia se encuentran otros lotes de mayor tamaño los cuales están en manos de colonos nacionales.

Por último, la legislación de 1874 permitió la asignación de importantes extensiones de tierras a particulares para establecer colonias con familias inmigrantes de Europa o Estados Unidos. Estas fueron las llamadas "concesiones" que en caso de la provincia de Cautin fueron las de Llaima, Budi, Lanín, Cautín, Queule y Nueva Etruria. Ninguna de estas concesiones, salvo la de la Empresa del Budi, se entregaron lotes de terrenos a familias colonas, situación que fue observada por la Comisión Parlamentaria en su informe de 1912.

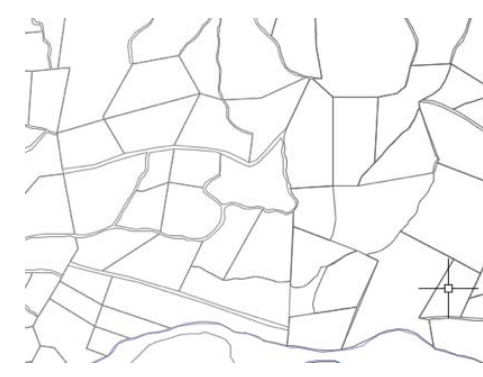

Trazado irregular. Radicación indígena

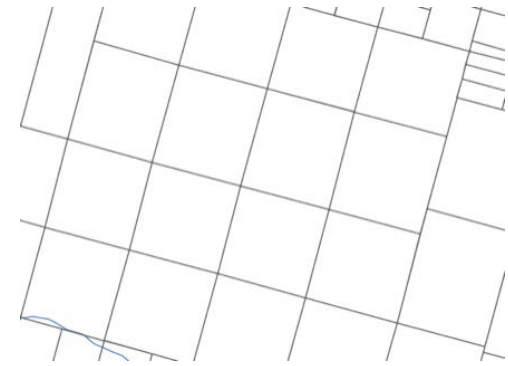

Trazado regular. Lotes entre 200 a 500 hectáreas

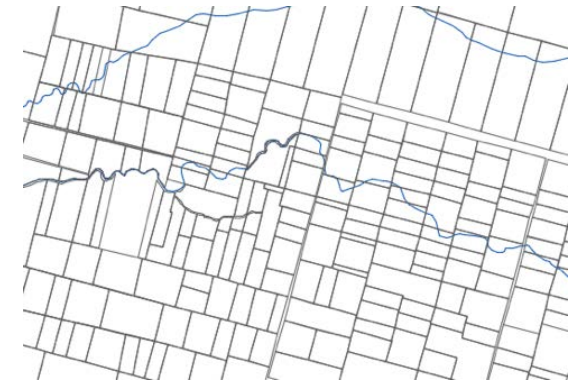

Trazado regular. Lotes inferiores a 200 hectáreas

Fig. 4. Formas de trazados. Elaboración propia

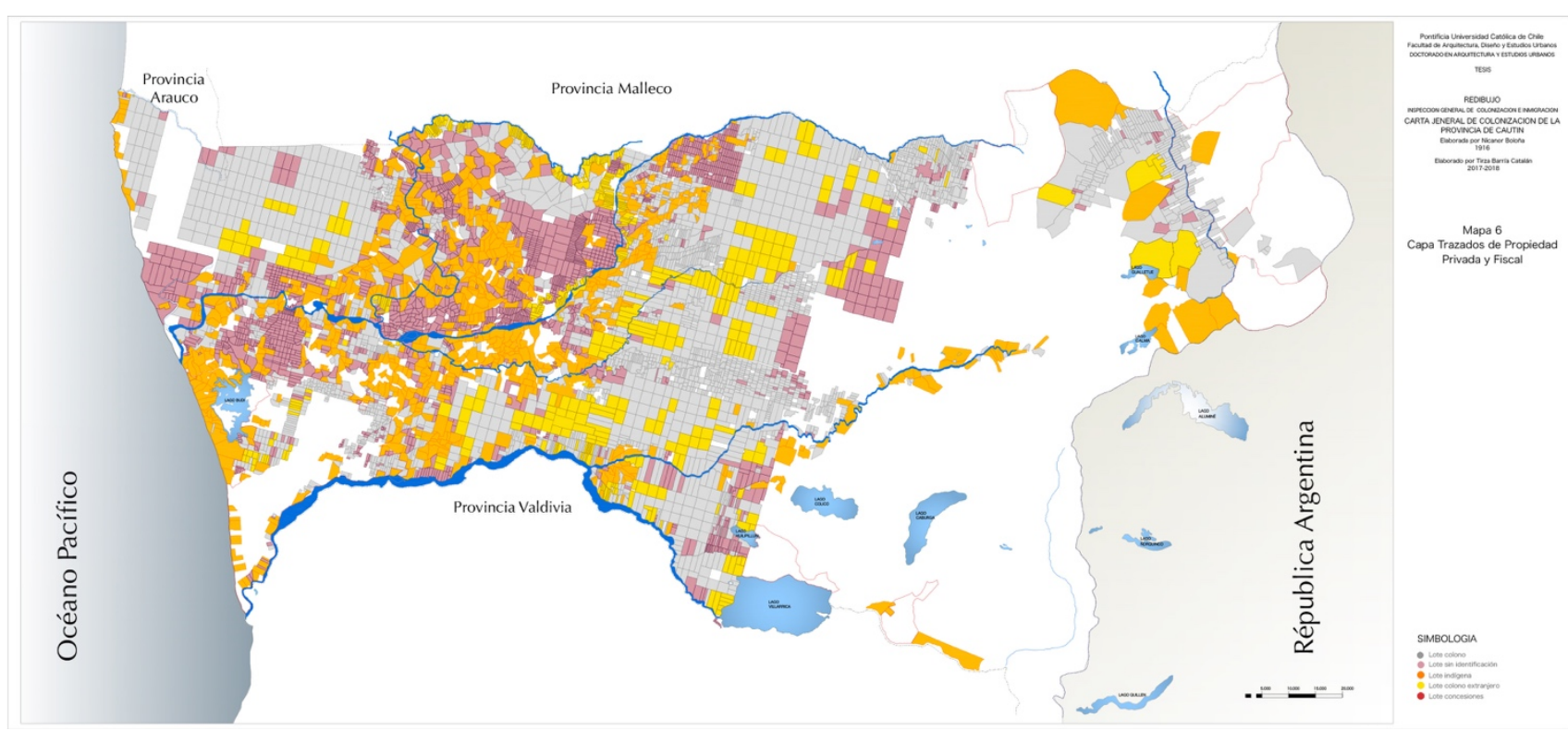

fig. 5 Mapa trazados lotes de propiedad privada y pública. Fuente: Elaboración propia a partir de los Cartas disponible en el Archivo Histórico de la Araucanía. 


\section{CONCLUSIONES}

En la denominada frontera araucana, se llevó a cabo la mensura del suelo, el remate de tierras para asentar la futura colonización nacional y extranjera y, la radicación mapuche. Esta forma de proceder determinó que la división del suelo - el trazado y su dimensión - estuviera condicionado al tipo de ocupante sea este mapuche o colono, determinado por la Ley del 4 de diciembre de 1866 y también a, la disponibilidad de tierras incorporadas al estado chileno, tras el avance militar de la "pacificación de la Araucanía".

Los dos mapas expuestos, tanto el de 1908 como el de 1916 exponen un estado del arte del territorio, por lo que se manifiesta una nueva forma de producción de conocimiento en donde la representación y escala del documento cartográfico resulta funcional para obtener una visión total de la envergadura y alcances de la colonización. Es decir orresponde a un catastro del territorio colonizado y radicado, que fija la propiedad de la tierra, a los cuales le precede en información y detalle el mapa de 1908.

La "Carta de 1916" es el resultado de la compilación de otros registros, y solo pueden dar cuenta de una territorialización avanzada y definitiva, cuyo proceso se había iniciado 50 años antes. Por otra parte, visibiliza los alcances logrados y fallidos de las decisiones políticas en cuanto a la implementación de un sistema de reparticiones de tierras, desde la escala de la ciudad hasta las grandes extensiones otorgadas a las concesiones de colonias, y los graves incumplimientos y arbitrariedades en los procesos de repartición

Esta Carta es una impresión de un modelo de ocupación en el territorio, lo que implica la especificidad del mecanismo. Es por tanto el encuentro entre una malla geometrizada de la urbanización y ruralización, donde aún la gestión del territorio y la ciudad, son saberes fragmentarios. Sin embargo, se buscará orden y control sobre la base de la ubicación especifica de los nuevos trazados urbanos, apoyados por una infraestructura que en el fondo persiguen el mismo objetivo dar cuenta de lo realizado en materia de colonización y ocupación a una escala de representación que permite visualizar a la provincia como un total.

Si bien las operaciones de repartición del suelo buscaron hacer desaparecer la ambigüedad de un territorio "vacío", por medio de trazados que impusieran un orden al territorio, las mensuras parciales confeccionadas para el remate de tierras determinaron la colonización de la zona. Ellas dan cuenta de formas de repartición las cuales apuntaban a la regularidad, articulación y continuidad del espacio territorial, en donde la repartición del territorio en algunos casos y dependiendo de la mensura, siguió la lógica de división y modulación geométrica, por otra parte, otros trazados se guiaron por formas topográficas de ríos y cerros dando forma a parcelas de bordes irregulares en su mayoría asociadas a tenencia mapuche.

En este sentido la mensura y repartición de tierras de la Araucanía, - el parcelario - sumado a la constitución de ciudades, fue vital para ordenar el territorio y fijar una trama coherente de relaciones entre el hombre y el territorio, cuya armonía dependía en parte de anular y ocupar desde un acto civilizatorio los espacios "vacíos" y tender a la homologación simétrica de ellos y prever su expansión.

Por último, no es menor mencionar que este mapa constituye un caso de estudio único e inédito de investigación en Chile, debido a que no existe hasta el momento, otro caso de registro territorial a escala provincial (salvo el elaborado para la provincia de Malleco en 1917) con el nivel de detalle y compilación de antecedentes relacionados con la tenencia de tierras, en donde el mapa da cuenta de una forma de representación del discurso político en un contexto marcado por la construcción del Estado y de las instituciones científicas, y por el desarrollo de instrumentos para la afirmación de la propiedad privada de la tierra y de la fiscalización del Estado. Por esta razón se busca situar su contexto de producción, y restituir sus conexiones con los marcos culturales y sociales que le dieron origen y que también resultaron definidos y modelados por unas prácticas técnicas que de ninguna manera fueron neutras. 


\section{BIBLIOGRAFIA}

ALVAREZ, Lily. "Catastro de propiedad en Chile: Origen y Evolución". Tesis de Doctorado en Geografía, Planificación territorial y Gestión ambiental, Universidad de Barcelona, 2014.

ANTIVIL, Wladimir. Dibujando la Araucanía. La construcción, la forma y el dominio de un territorio. Universidad Politécnica de Cataluña. Tesis doctoral. 2018.

AYLWIN, José. "Estudios sobre tierras indígenas de la Araucanía: Antecedentes histórico legislativo 18501920". Temuco, Chile: Instituto de Estudios Indígenas, Universidad de La Frontera. Serie Documentos No 3 , 1995.

COMISIÓN PARLAMENTARIA DE COLONIZACIÓN. Informes, proyectos de Ley, actas de las sesiones y otros antecedentes. Santiago de Chile: Imprenta y Litografía Universo. 1912

ESPINOZA, Enrique. Jeografía Descriptiva de la República de Chile. Santiago: Editorial Imprenta, Litografía y Encuadernación Barcelona. 1903.

FAVELUKES, Graciela. Mapas catastrales y control territorial: Mensuras, mapas, atlas. En XIV Jornadas Interescuelas/Departamentos de Historia. Disponible en http://cdsa.aacademica.org/000-010/934.pdf. 2013.

FLORES, Jaime; AZÓCAR, Alonso. Mapas para el Estado. La representación de la Araucanía: 1836 -1916. Scripta Nova. Revista Electrónica de Geografía y Ciencias Sociales. [En línea]. Barcelona: Universidad de Barcelona, vol. XXI, n 562. [ISSN: 1138-9788]. 2017.

GONZÁLEZ, José Ignacio. Primeros levantamientos cartográficos generales de Chile con base científica: los mapas de Claudio Gay y Amado Pissis. Revista Geografía Norte Grande № 38, p. 21-44. 2007.

GONZÁLEZ, José Ignacio; BERMEDO, Patricio. Cartografía de la transformación de un territorio: La Araucanía 1852-1887. Revista Geografía Norte Grande No 54, 2013. pp. 179-198

GUEVARA, Tomás. La ocupación de la Araucanía. En Historia de la civilización araucana, capítulo V. Santiago, Chile: 1902.

JACKSON TURNER, Frederick. La frontera en la historia americana San José, Costa Rica: Universidad Autónoma de Centroamérica, 1986.

MÉNDEZ, L. La organización de los parlamentos de indios en el siglo XVIII. En S. Villalobos, et al. Relaciones fronterizas en la Araucanía. Santiago, Chile: Ediciones Universidad Católica de Chile. 1982.

NAVARRO, Leandro. Crónica militar de la conquista y pacificación de la Araucanía desde el año 1859 hasta su completa incorporación al territorio nacional. Santiago, Chile: Imprenta y Encuadernación Lourdes, 1909.

PARCERISA, Josep y BUSQUETS, Joan. Instruments de projectació de la Barcelona suburbana. Annals d'arquitectura, №. 2, 1983.

SAAVEDRA, Cornelio. Documentos relativos a la ocupación de Arauco que contiene los trabajos practicados desde 1861 a la fecha. Santiago. Imprenta de La Libertad. 1870

VILLALOBOS, Sergio. Tres siglos y medio de vida fronteriza y Tipos fronterizo en el ejército de Arauco. En S. Villalobos et al., Relaciones fronterizas en la Araucanía. Santiago, Chile: Ediciones Universidad Católica de Chile, 1982. 